\title{
A simple mathematical model for collagen fibril organization in normal and keratoconic corneas
}

Peter M. Pinsky ${ }^{1}$, Yanhui Ma ${ }^{2}$, Yunjae Hwang ${ }^{1}$, Sally Hayes ${ }^{2}$, Keith M. Meek ${ }^{2}$

${ }^{1}$ Department of Mechanical Engineering, Stanford University, Stanford, California, USA, ${ }^{2}$ School of Optometry and Vision Sciences, Cardiff University, Cardiff, UK

Key words: anisotropy, collagen fibril organization, cornea, keratoconus, $\mathrm{x}$-ray scattering

\section{Background and purpose}

Wide-angle $x$-ray scattering techniques have been used to provide quantitative information about the angular distribution and preferred orientation of collagen fibrils throughout the corneaMeek, Boote [1]. Using this technique, it has been shown that collagen fibrils in the human cornea exhibit a preferential alignment in the inferiorsuperior and nasal-temporal directionsAghamohammadzadeh et al. [2]. However, this arrangement is often seen to be absent or disturbed in cases of keratoconusHayes et al. [3] and Meek et al. [4], a dystrophy in which the cornea becomes progressively thin and weak, resulting in the appearance of a cone-shaped cornea.

The elasticity of stromal tissue depends on the distribution of collagen fibrils throughout the cornea, and preferential fibril alignments imply that the tissue is mechanically anisotropic. Finite element models for corneal biomechanics can account for this mechanical anisotropy by employing a fibril angular orientation probability function based on x-ray scattering patternsCheng et al. [5]. However, the scattering patterns are obtained only at a discrete set of collection points, whereas finite element models need a continuous representation over the cornea. To address this need, a mathematical modeling approach is proposed that converts the $x$-ray data at the collection points into a single continuous function over the corneaMa et al. [6]. This representation of the x-ray data is necessary for finite element analysis, as well as valuable for quantitatively analyzing and comparing fibril organization in normal and keratoconic corneas.

Correspondence: Peter M. Pinsky, Department of Mechanical Engineering, Stanford University, Stanford, CA 94305-4040, USA.

E-mail: pinsky@stanford.edu 


\section{Methods}

The modeling approach uses two fitting steps, both based on least squares. First, the $x$-ray scattering data at each collection point is modeled as a continuous function of the $\mathrm{x}$-ray scan angle, $\theta$, using the following representation:

$$
s_{1}(\theta ; \mathbf{p})=a_{1} \cos ^{n_{1}}\left(\theta-\beta_{1}\right)+a_{2} \sin ^{n_{2}}\left(\theta-\beta_{2}\right)+c,
$$

where $\mathbf{p}=\left\{a_{1}, a_{2}, \beta_{1}, \beta_{2}, c\right\}$ are five parameters determined from a non-linear least squares problem (trials indicated that $n_{1}=n_{2}=6$ in all cases). In this way, $\mathrm{x}$-ray scattering patterns at each collection point are reduced to five parameter values. In the second fitting step, the five parameters at each collection point are given a continuous representation over the corneal unit disk using Zernike basis functions, such that:

$$
\begin{aligned}
s_{2}\left(r^{\prime}, \varphi ; \theta\right) & =\left(\sum_{i=1}^{p_{a_{1}}} \widehat{\kappa}_{a_{1}}^{i} Z_{i}\left(r^{\prime}, \varphi\right)\right) \cos ^{6}\left(\theta-\sum_{i=1}^{p_{\beta_{1}}} \widehat{\kappa}_{\beta_{1}}^{i} Z_{i}\left(r^{\prime}, \varphi\right)\right) \\
& +\left(\sum_{i=1}^{p_{a_{2}}} \widehat{\kappa}_{a_{2}}^{i} Z_{i}\left(r^{\prime}, \varphi\right)\right) \sin ^{6}\left(\theta-\sum_{i=1}^{p_{\beta_{2}}} \widehat{\kappa}_{\beta_{2}}^{i} Z_{i}\left(r^{\prime}, \varphi\right)\right)+\sum_{i=1}^{p_{c}} \widehat{\kappa}_{c}^{i} Z_{i}\left(r^{\prime}, \varphi\right),
\end{aligned}
$$

where $\left(r^{\prime}, \varphi\right)$ are polar coordinates on the unit disk and the five summations are expansions for the five parameters in $\mathbf{p}$ using Zernike basis functions $Z_{i}\left(r^{\prime}, \varphi\right)$. The coefficients $\widehat{\kappa}_{p}^{i}$, for $p \in \mathbf{p}$, are obtained by solving a linear least squares problem. To prevent over-fitting and to maximize interpolation accuracy, multiple applications of ten-fold cross-validationGeisser [7] were used to establish the optimal radial order of the Zernike expansions in Equation (2).

\section{Results}

Data from four normal post-mortem corneas and four severe keratoconus corneal disks (removed during routine transplant surgery), were analyzed. X-ray scattering data were collected over the entire cornea in the case of the healthy samples and over a central $8 \mathrm{~mm}$ corneal disk in the case of the keratoconic samples. The accuracy of Equation (1) in reproducing the raw $\mathrm{x}$-ray scattering plots for normal and keratoconic corneas at three typical collection points is illustrated in Figure 1. Fittings based on Equation (1) for both healthy and keratoconic corneas were obtained with high precision in all cases. In the second fitting step based, on Equation (2), ten-fold cross-validation studies determined that the maximum Zernike radial order is casedependent; for normal corneas the optimal radial order was near 10, while for keratoconic corneas the optimal order was near 15 . Figure 2 (row 1) illustrates the result of 
the second fitting, showing the contours of the $\beta_{1}$ and $\beta_{2}$ coefficients (phase-shift of preferred orientations) for a normal and a keratoconic cornea. By integrating Equation (2) over $\theta \in[0,2 \pi]$, maps of total collagen mass and aligned collagen mass can be obtained. This is illustrated in Figure 2 (row 2), which contrasts mass contours for a normal and a keratoconic cornea.
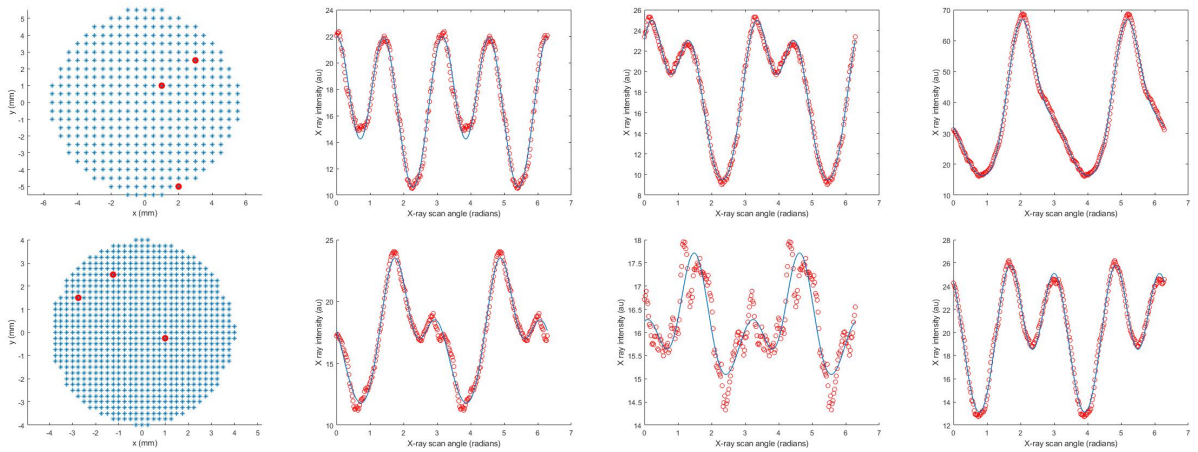

Fig. 1. Comparison of $x$-ray scattering data (red circles) and fitting by Equation (1) (blue curve). (Top row, left to right) Collection points and fit at three selected points for a healthy cornea. (Bottom row, left to right) Collection points and fit at three selected points for a keratoconic cornea.
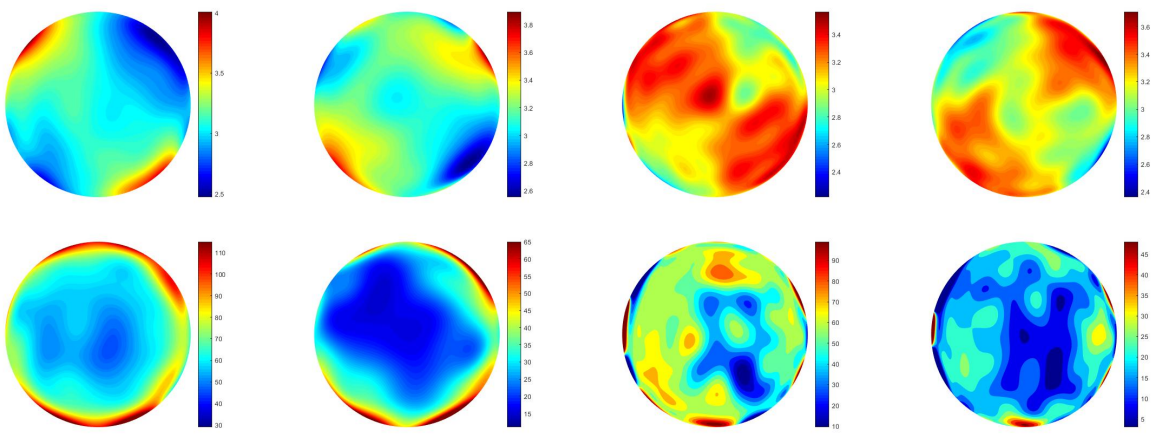

Fig. 2. Selected results from the second fitting based on Equation (2). (Top row, left to right) Contours of $\beta_{1}$ and $\beta_{2}$ for a normal cornea and for a keratoconic cornea. (Bottom row, left to right) Contours of predicted total collagen mass and aligned (anisotropic) collagen mass for a normal cornea and for a keratoconic cornea. 


\section{Conclusions and future perspectives}

The proposed mathematical approach is based on two sequential fittings. The first fitting, based on the Fourier-type form given by Equation (1), proved to be accurate for every cornea analyzed. The Zernike expansion of the coefficients, expressed by Equation (2), also proved to have high predictive accuracy, but requires strict adherence to the optimal radial order as determined by the cross-validation studies.

Total and aligned collagen mass contours of the normal corneal pair have been previously published and agree with contours found from the model, providing a measure of the overall accuracy of the model. While keratoconic corneas may appear to exhibit great irregularity in collagen angular orientation, it is significant that Equation (1) models the x-ray data of both healthy and keratoconic corneas with high precision, suggesting that keratoconic collagen organization, even in severe cases, may result from a redistribution of the normal arrangement, possibly by lamellar slippageMeek et al. [4]. This conclusion is further reinforced by considering, for example, the contours of $\beta_{1}$ and $\beta_{2}$ shown in Figure 2 . The normal cornea shows values that agree with preferred orientations in the inferior-superior and nasal-temporal directions; likewise, the keratoconic cornea has an essentially similar pattern except that it has become profoundly diffuse.

All completed tests have confirmed that the proposed modeling approach can accurately describe collagen organization in normal and keratoconic corneas. Next steps include implementation in a finite element analysis code and further geometric analysis to map the model onto the corneal sphere in order to reveal an improved interpretation of collagen organization.

\section{Acknowledgements}

This work has been supported by a Medical Research Council UK Programme Grant MR/K000837/1 awarded to Keith M. Meek. We thank Diamond Light Source (UK) for access to beamline 102 for the collection of x-ray scattering data, Mr. Stephen Tuft for the provision of keratoconus corneas, and the UK National Health Service Blood and Transplant for the provision of healthy donor corneas. A travel grant awarded to Yanhui Ma from the PGR International Experience Fund, Cardiff University (UK), is acknowledged.

\section{References}

1. Meek K, Boote $\mathrm{C}$. The use of X-ray scattering techniques to quantify the orientation and distribution of collagen in the corneal stroma. Prog Retin Eye Res. 2009;28(5): 369-392.

2. Aghamohammadzadeh $\mathrm{H}$, Newton R, Meek K. X-ray scattering used to map the preferred collagen orientation in the human cornea and limbus. Prog Retin Eye Res. 2004;12(2): 249-256.

3. Hayes S, Boote C, Tuft S, Quantock A, Meek K. A study of corneal thickness, shape and collagen organisation in keratoconus using videokeratography and X-ray scattering techniques. Exp Eye Res. 2007;84(3): 423-434. 
4. Meek K, Tuft S, Huang Y, Gill P, Hayes S, Newton R. Changes in collagen orientation and distribution in keratoconus corneas. Invest Ophthalmol Vis Sci. 2005;46(6): 1948-1956.

5. Cheng $X$, Petsche $S$, Pinsky $P$. A structural model for the in vivo human cornea including collagenswelling interaction. JR Soc Interface. 2015;12(109): doi: 10.1098/rsif.2015.0241.

6. Ma Y, Hwang Y, Hayes S, Meek K, Pinsky P. A simple mathematical model for collagen fibril organization in normal and keratoconic corneas. Proceedings, ARVO Annual Meeting, Baltimore, MD, 2017.

7. Geisser S. Predictive inference. Chapman and Hall, 1993. 